\title{
Mechanistic insights into Bardet-Biedl syndrome, a model ciliopathy
}

\author{
Norann A. Zaghloul ${ }^{1}$ and Nicholas Katsanis ${ }^{1,2,3}$
}

${ }^{1}$ McKusick-Nathans Institute of Genetic Medicine, ${ }^{2}$ Wilmer Eye Institute, and ${ }^{3}$ Department of Molecular Biology and Genetics, Johns Hopkins University School of Medicine, Baltimore, Maryland, USA.

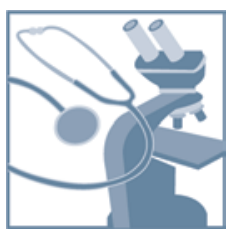

Bardet-Biedl syndrome (BBS) is a multisystemic disorder typified by developmental and progressive degenerative defects. A combination of genetic, in vitro, and in vivo studies have highlighted ciliary dysfunction as a primary cause of BBS pathology, which has in turn contributed to the improved understanding of the functions of the primary cilium in humans and other vertebrates. Here we discuss the evidence linking the clinical BBS phenotype to ciliary defects, highlight how the genetic and cellular characteristics of BBS overlap with and inform other ciliary disorders, and explore the possible mechanistic underpinnings of ciliary dysfunction.

Bardet-Biedl syndrome (BBS; MIM 209900) is a genetic disorder characterized by defects in multiple organ systems, and the estimated prevalence ranges from one in 160,000 in northern European populations $(1,2)$ to as high as one in 13,500 in Kuwait and Newfoundland $(3,4)$. Multiple lines of evidence have indicated that the BBS phenotype is largely a consequence of ciliary dysfunction. These have included the localization of most BBS proteins to the basal body and the ciliary axoneme, the restricted evolution of $B B S$ genes in ciliated species, and their expression in ciliated cells. More recently, direct evidence has also been obtained, including the demonstration of structural and functional ciliary defects in cells and tissues lacking BBS proteins. Collectively, these findings highlight the importance of cilia to the development and homeostasis of a broad range of tissues. Moreover, understanding of the phenotypic overlap between BBS and other ciliary disorders has drawn attention to the similarities between traditionally discrete clinical disorders, such as nephronophthisis (NPH), Joubert syndrome (JBTS), and Meckel-Gruber syndrome (MKS), offering the possibility that mechanistic insights gleaned from some ciliopathies might inform the etiopathology of other/all ciliopathies. Here we review some of the major aspects of the BBS phenotype that overlap with other syndromic ciliopathies and discuss emerging mechanistic models that potentially underlie the observed pathology.

\section{Clinical synopsis of BBS}

Laurence and Moon first reported this disorder in 1866 with a description of a child with obesity, visual impairment, and mental disabilities (5), and later reports by Bardet and Biedl described patients with similar features, in addition to polydactyly and hypogenitalism $(6,7)$. These remain the characteristic features of the disorder. However, as our understanding of the phenotype has

Conflict of interest: The authors have declared that no conflict of interest exists. Nonstandard abbreviations used: ARPKD, autosomal recessive PKD; BBS, BardetBiedl syndrome; CC, connecting cilium; IFT, intraflagellar transport: IS, inner segment; GLI, glioma; JBTS, Joubert syndrome; LCA, Leber congenital amaurosis; MKS, Meckel-Gruber syndrome; NPH, nephronophthisis; ONL, outer nuclear layer; OS, outer segment; PCM, pericentriolar material; PCP, planar cell polarity; PKD, polycystic kidney disease; POMC, proopiomelanocortin; PTCH1, Patched1; Shh, Sonic hedgehog; SMO, Smoothened; stbm, strabismus; ZPA, zone of polarizing activity. Citation for this article: J. Clin. Invest. 119:428-437 (2009). doi:10.1172/JCI37041. progressed, previously unrecognized features have been included, whereas the inclusion of others as hallmarks of the disorder, such as mental retardation, has been challenged (8).

The clinical phenotype (and its variability) has been discussed extensively (9). Briefly, there are six major features that are considered the hallmarks of the disorder $(2,9)$ based on their prevalence in the patient population: retinal degeneration, obesity, hypogonadism, polydactyly, renal dysfunction, and mental retardation. Several minor features have also been associated with BBS, including neurological impairment, speech deficits, craniofacial abnormalities, hearing loss, diabetes mellitus, metabolic defects, cardiovascular abnormalities, hepatic defects, and Hirschsprung disease (9). Recently, the establishment of the ciliary link for BBS has led to the identification of previously unrecognized phenotypes that include anosmia and defects in thermosensory and nociceptive sensation $(10,11)$.

\section{The genetic cause(s) of BBS}

BBS is a disorder of locus and allelic heterogeneity. It is typically inherited in an autosomal recessive fashion, under which model mutations in 14 loci (BBS1-12, meckel syndrome 1 [MKS1], centrosomal protein $290 \mathrm{kDa}$ /nephronophthisis 6 [CEP290/NPHP6]) have been identified (ref. 12 and refs. therein; Supplemental References S1-S15; supplemental material available online with this article; doi:10.1172/JCI37041DS1). The contribution of each locus to total mutational load varies across populations, as does mutant allele frequency (Figure 1). For example, in patients of Northern European descent, $B B S 1$ and $B B S 10$ contribute approximately $40 \%-50 \%$ of known mutations (13-15), most of which are contributed by two alleles, the M390R allele in BBS1 and the C91fsX95 allele in BBS10. In families of Arab ancestry, however, the frequency of C91fsX95 remains the same as in individuals of Northern European descent, suggesting that this mutation arose early during the human diaspora, while the M390R allele is thought to have arisen in an ancient haplotype (13) that is largely absent from non-European populations. At the other extreme, most other loci are rare contributors; for example, there is only a single documented family with mutations in each of BBS11, MKS1, and CEP290 $(12,16)$.

BBS is also typified by profound inter- and intrafamilial clinical variability, which can be explained in part by the presence of second-site modifiers. In some families, loss of function at a primary 


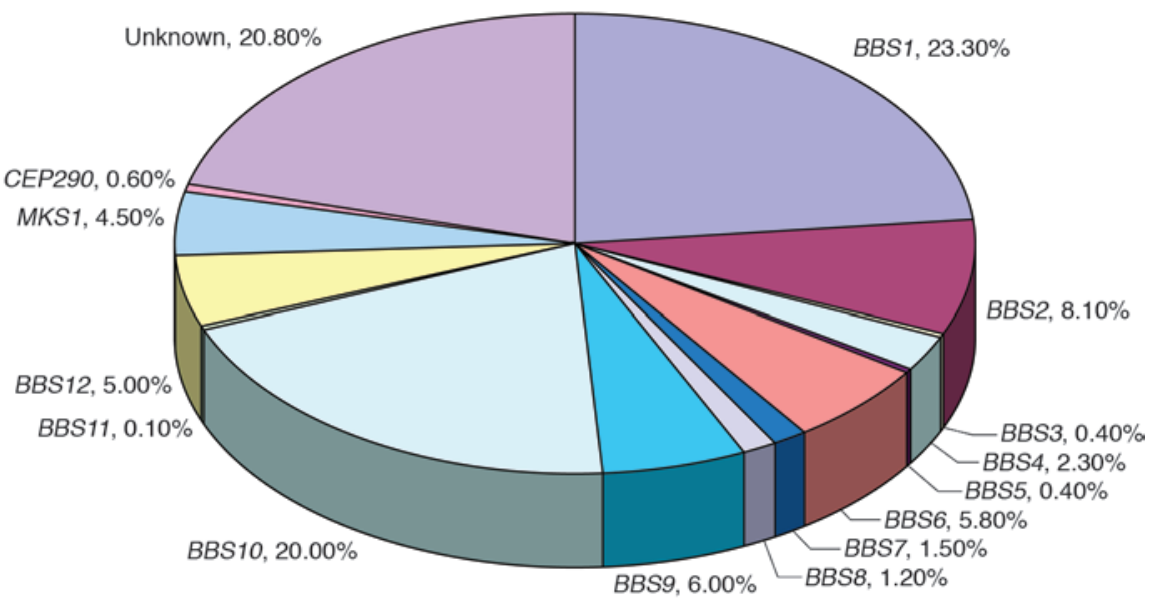

\section{Figure 1}

Contribution of each BBS gene to total mutational load. Graph of the percentage contribution of each known BBS gene to BBS cases. Modified and updated from Human Molecular Genetics (14). locus combines with haploinsufficiency at a second BBS locus to cause disease $(17,18)$, while in other families, second-site mutations likely serve as modifiers of expressivity $(12,15,19-21)$. The ability of each BBS locus to contribute alleles that can modify the effect of mutations in other BBS genes suggested a common pathway and potentially the existence of a macromolecular complex under the "poison" hypothesis - according to which the presence of mutant subunits in a heterodimer or multisubunit complex prevents proper functioning of the entire assembly (22) (S16). Experimental evidence linking BBS proteins to ciliary function (23-28) and biochemical data suggesting that at least seven BBS proteins can interact with each other to form a complex (28) support and extend this notion.

Linking BBS to ciliary dysfunction. The first evidence that BBS is a defect of ciliary function was obtained from the identification of $B B S 8$ (23). Of the three families with loss-of-function mutations in this transcript, one patient was also diagnosed with situs inversus, a defect of left-right axis determination in which the major visceral organs are reversed from their normal positions, a common outcome of defects in nodal cilia (29). Moreover, BBS8 was shown to colocalize with $\gamma$-tubulin in centrioles, and, critically, transcriptional GFP constructs of all recognizable bbs genes in the nematode Caenorhabditis elegans demonstrated restricted expression in ciliated sensory neurons (23). Both the cloning of additional BBS genes and the retrospective study of previously identified molecules extended these findings and transitioned the research focus to the dissection of the molecular defect. All BBS proteins studied to date localize primarily to centrosomes, basal bodies, or cilia (23-28), and recent evidence also suggests that the BBS complex can associate with the RAB8 GDP/GTP exchange factor to promote trafficking of vesicles to the cilium, a process necessary for the elongation of the ciliary membrane (28).

In addition to cellular localization studies, results from animal models, most notably C. elegans, showed that bbs 8 may be involved in the regulation of the RFX transcription factor, which regulates expression of ciliary protein-encoding genes (23). These findings suggest an important role for BBS proteins in ciliary function, and these proteins have been associated with ciliogenesis and intraflagellar transport (IFT), the motor-dependent trafficking of cargo along the cilium (S17). This was demonstrated when loss of bbs 7 and bbs 8 in C. elegans produced structural defects in cilia as well as the mislocalization of IFT proteins, such as IFT88, along the ciliary axoneme (30). A subsequent study showed that these proteins likely function to stabilize the association of two IFT motor proteins, kinesin and OSMotic avoidance abnormal 3 (OSM-3), to ensure the proper rate and function of IFT transport (31).

In addition to playing a key role in transport of IFT proteins, there is evidence that BBS proteins might also take part in other forms of transport. The most notable example is melanosome transport. Results from zebrafish models indicate that loss of BBS proteins results in suppression of melanosome shuttling from the cell periphery to the perinuclear region, a process termed retrograde transport (32). Because this process is mediated by the microtubule-based motor proteins kinesin II and dynein in nonciliated cells, this finding suggests that the BBS proteins may also be involved in non-ciliary-related microtubule-based transport.

\section{Cilia in disease}

Since the first observation of cilia in the renal epithelium and the thyroid gland (S18), these organelles are now understood to be present in numerous mammalian cell types. Projecting from the apical surface of the plasma membrane and tethered to the basal body, primary cilia typically consist of nine microtubule doublets around the periphery extending from the basal body. A subset of cilia, known as motile cilia, contain a central microtubule pair that is thought to impart additional function (S19). The extensive presence of cilia in the vertebrate body plan explains the wide range of phenotypes associated with defects in their structure and/or function, and defects in both primary and motile cilia contribute to disease. To date, cell types known to be impacted by ciliary dysfunction include renal and retinal tissues as well as the neural tube, developing limbs, pancreas, liver, spleen, bone, and several parts of the CNS (33).

Defects that impact the function of cilia (directly or indirectly) have been demonstrated in a number of diseases, including polycystic kidney disease (PKD), NPH, Alstrom syndrome (AS), and BBS. The common causality and genetic overlap has led to the grouping of these discrete clinical entities to a unified entity, the ciliopathies (33), while the availability of integrated gene/protein sets and databases for ciliary proteins (ref. 34 and refs. therein) is facilitating both the identification of new genes mutated in these disorders and the recognition of additional clinical entities as ciliopathies. 


\section{Table 1}

Clinical phenotypes associated with ciliopathies

\begin{tabular}{|c|c|c|c|c|c|c|c|c|c|}
\hline & MKS & BBS & JBTS & JATD & OFD1 & MKKS & SLS & NPH & LCA \\
\hline Retinopathy & + & + & + & - & - & - & + & + & + \\
\hline Polydactyly & + & + & + & - & + & + & - & - & - \\
\hline Kidney disease & + & + & + & + & + & - & + & + & - \\
\hline Situs inversus & + & + & + & - & - & - & + & - & - \\
\hline Mental retardation/developmental delay & + & + & + & - & + & - & - & + & + \\
\hline Hypoplasia of cerebellum & + & + & + & - & + & - & - & + & - \\
\hline Hydrometrocolpos & - & + & - & - & - & + & - & - & - \\
\hline Obesity & - & + & + & - & - & - & - & - & - \\
\hline Hepatic dysfunction & + & + & + & - & - & - & + & + & - \\
\hline
\end{tabular}

JATD, Jeune syndrome; OFD1, orofaciodigital syndrome 1; MKKS, McKusick-Kaufman syndrome; SLS, Senior-Loken syndrome.

\section{BBS as a model ciliopathy}

The characteristic features of BBS have also been observed in a number of other ciliopathies, making BBS a useful model disorder. This overlap can be observed at several levels: phenotype, contribution of pathogenic alleles of ciliopathy genes across the entire spectrum of clinical entities, protein colocalization, and participation in common signaling pathways.

Phenotypic overlap. Compromised retinal and kidney function is observed across a range of ciliopathies due to defects in photoreceptor and renal cilia, including the more severe pleiotropic disorders such as MKS and JBTS, as well as disorders affecting fewer organ systems, such as Senior-Loken Syndrome (SLS), NPH, and Leber congenital amaurosis (LCA) (Table 1). In other cell types, dysfunction leads to developmental abnormalities, such as polydactyly and mental retardation. Such similar phenotypes have been observed not only in BBS but also in other ciliopathies, including MKS, JBTS, orofaciodigital syndrome 1 (OFD1), McKusick-Kauffman syndrome (MKKS), NPH, and LCA and have been discussed extensively elsewhere (33).

Genetic overlap. Emerging data suggest that ciliopathy-associated genes have the capacity to contribute pathogenic alleles to multiple ciliopathies (Table 2). For example, genes that cause MKS, such as MKS1 (35) and MKS3 (36), have been shown recently to contribute both causative and modifying alleles to BBS patients (12). Likewise, the most recently identified BBS gene, CEP290/NPHP6, had been linked previously to a number of other ciliopathies, including MKS, JBTS, SLS, NPH, and LCA (37) (S20-S22). BBS genes have also been causally associated with other disorders, exemplified by the identification of heterozygous mutations in BBS2 and BBS4 in fetuses with an MKS-like phenotype (38). Such an overlap is not unique to BBS within the ciliopathy disease group, where both the quality and quantity of alleles can impact pleiotropy and severity. Hypomorphic mutations in NPHP3 cause NPH, whereas null mutations in the same gene cause MKS (39); meanwhile the presence of bona fide pathogenic mutations at two NPHP loci has also been described (40), and these have been postulated to modulate the expressivity of the disease phenotype (41).

$B B S$ and ciliary complexes. All BBS proteins investigated to date localize primarily to the basal body of mammalian cells and in the transition zone of ciliated sensory neurons of C. elegans (23-28). Not surprisingly, the subcellular distribution of the BBS proteins overlaps with that of a host of other ciliopathy-associated molecules, and in some instances biochemical interactions between these mol- ecules and BBS proteins have also been observed. BBS4, BBS6, and CEP290/NPHP6 each associate with the pericentriolar material (PCM) $(26,42-44)$, an amorphous network of proteins that surrounds the centrosome (45). Furthermore, the BBS proteins can form a complex with each other in cell culture that also interacts with PCM1, the main component of the PCM (28). The interaction of BBS proteins with PCM1 is significant, because, at least in the case of CEP290, it is necessary for PCM1 localization and for the proper organization of the cytoplasmic microtubule network (44).

The association of MKS1 with BBS (12) provided further support for the biochemical overlap of BBS and MKS. Additionally, MKS1, which localizes to the basal body, interacts with MKS3, which localizes to the cilium and the plasma membrane (46). This interaction directly links BBS and MKS at the protein level, suggesting that their cellular dysfunction is regulated by defects in overlapping pathways. There is evidence that different complexes may be involved in the overlapping regulation of ciliary function by BBS and MKS proteins. For example, ablation of the C. elegans MKS1 ortholog $x b x 7$, or other proteins bearing the B9 domain common to a number of ciliary proteins, has no overt effect on ciliary morphology; however, the addition of a mutation in $n p h 4$, the homolog of which is mutated in NPH patients, results in defective cilia (47).

\section{Molecular mechanisms underlying ciliary phenotypes}

An understanding of the molecular defects in BBS has provided insight into the defects of other ciliopathies. At the same time, our expanding understanding of the roles of cilia is both informing the etiopathology of the human phenotypes and unmasking novel, often subtle, clinical defects in patients with BBS and other ciliopathies.

Retinal defects. BBS patients manifest a progressive retinal dystrophy of the photoreceptors, sometimes with early macular involvement (48). Mice in which $B b s$ genes are modified model this phenotype to a large extent, albeit not at full penetrance. Bbs1-, Bbs2-, $B b s 4$-, and Bbs6-null mice, as well as a knock-in model of a common BBS1 mutation, M390R, display moderate loss of outer nuclear layer (ONL) retinal tissue by 6-8 weeks of age, which correlates, at least in Bbs2- and Bbs4-null mice, with increased cell death (49-53). More detailed observations of photoreceptor structure in BBS retinas indicate that, though lamination of the retina is intact, photoreceptor integrity is disrupted in the outer layers (54). This is morphologically similar to the degeneration observed in patients with Alstrom syndrome (55) and LCA (56). 
Table 2

Overlap of ciliary genes contributing to ciliopathies

\begin{tabular}{lccccccc} 
& MKS & BBS & JBTS & MKKS & SLS & NPH & LCA \\
BBS2 & + & + & - & - & - & - & - \\
BBS4 & + & + & - & - & - & - & - \\
BBS6/MKKS & + & + & - & + & - & - & - \\
MKS1 & + & + & - & - & - & - & - \\
MKS3/TMEM67 & + & + & + & - & - & - & - \\
NPHP1 & - & - & + & - & + & + & - \\
NPHP2/INVS & - & - & - & - & + & + & - \\
NPHP3 & + & - & - & - & + & + & - \\
NPHP4 & - & - & - & - & + & + & - \\
NPHP5 & - & - & - & - & + & + & - \\
CEP290/NPHP6 & + & + & + & - & + & + & + \\
RPGRIP1L & + & - & + & - & - & - & - \\
CC2D2A & + & - & + & - & - & - & - \\
\hline
\end{tabular}

signaling, and one might expect that an attempt of the photoreceptor to reenter the cell cycle will also lead to apoptosis (S24).

Obesity. The penetrance of the obesity phenotype in patients suggests that the BBS proteins play a fundamental role in energy regulation. However, little is known about their actual role(s). $B b s$-knockout mice and the Bbs1-M390R knock-in display obesity phenotypes and increased feeding behavior (49, 50, 52). Additionally, Bbs4- and Bbs6null mice exhibit increased blood pressure and increased levels of the circulating hormone leptin $(50,51)$. The Bbs1-M390R mouse is also hyperphagic and hyperleptinemic, with reduced locomotor activity (53). The role of leptin was further linked to the BBS phenotype when a recent study showed not only that Bbs2-, Bbs4-, and Bbs6-null mice had increased leptin levels but that this may be a result of resistance to exogenous leptin (70).

Some reports have suggested that photoreceptor cell death might be due to an underlying defect in the function of the connecting cilium (CC), a structure that links the inner segments (ISs) to the outer segments (OSs) of photoreceptors. In addition to the localization of at least one BBS protein, BBS8, to the CC (23), Bbs2-, Bbs4-, and Cep290/Nphp6-mutant mice show rhodopsin staining in the IS, suggested to reflect defective transport across the CC (49, $57,58)$. This is accompanied by an increase in the expression of stress response genes and apoptotic activity in Bbs4 mutants (59).

Some data are consistent with a transport defect, offering at least two mechanistic possibilities. Based on primarily a tissue culture model, it has been demonstrated that some BBS proteins are important for vesicular transport. The complex formed by several BBS proteins, the BBSome, associates with the GDP/ GTP exchange factor Rab8, which allows for vesicle trafficking to the base of the cilium (28). Consistent with a possible vesicular model of photoreceptor degeneration, Rab8 mutants display an accumulation of rhodopsin-carrying vesicles at the base of the CC (60). Additionally, localization of Rab8 to the cilium is facilitated by PCM1 (44).

A second possibility may be a defect in IFT across the CC. Mice and zebrafish with mutations in a number of Ift genes, including Ift88, Ift172, Ift52, and Ift57, exhibit a similar loss of photoreceptors (61-64). IFT proteins localize to the basal body in the IS and along the axoneme in the OS (65) and are necessary for IFT transport across the photoreceptor cilium (64); failure of this transport results in cell death (64).

At the same time, some observations are difficult to reconcile with a trafficking model. Rhodopsin and other IS proteins can translocate to the OS through other, CC-independent mechanisms (reviewed in ref. S23). Given the involvement of the cilium in a variety of signaling pathways, it is important to consider other possibilities. For example, BBS4, and probably other BBS proteins, such as BBS11, are involved in proteasomal-mediated degradation (66). Although it is not yet known whether rhodopsin degradation is affected in $B b s$ mutants, it is plausible that reduced proteasome activity might lead to the accumulation of material in the IS, which can then trigger ER stress and, eventually, apoptosis.

Alternatively, defective Wnt and Sonic hedgehog (Shh) signaling have each been associated with ciliary dysfunction (66-69). It is interesting to note that cyclin D1 is a known target of $\beta$-catenin
The role of neuronal and hormonal cues in regulation of feeding was further explored in Bbs2- and Bbs4-null mice where the neuronal ciliary localization of the $G$ protein-coupled receptor melanin-concentrating hormone receptor 1 (MCHR1), which regulates feeding behavior, was perturbed (71). Consistent with this finding, genetic screens of the C. elegans mutant tub1, an ortholog of the Tubby gene, indicate that alleles of a fat storage gene, kat 1 , may be involved in the obesity phenotype, and screens of kat 1 mutants indicate that $b b s 1$ may be perturbed in ciliated neurons that sense nutrient levels (72). The role of BBS proteins in the regulation of feeding may be related to IFT, as animals with knockout of either Ift88 or Kif 3 a were obese, with increased insulin and leptin levels, and these phenotypes may be specific to defects in hypothalamic neurons responsible for regulation of feeding $(73,74)$. Loss of cilia specifically in proopiomelanocortin (POMC) neurons by POMC:Cre deletion results in an increase in weight and adiposity, though these increases were not as severe as those seen as a result of systemic ablation $(73,74)$. These findings support a role for cilia in hypothalamic neurons in the brain's mediation of feeding behavior by interpretation of signals from various organs transmitted to the CNS, including hormonal satiety cues such as leptin and insulin. Leptin excites POMC neurons in the presence of high glucose levels to signal reduced food intake (75). If POMC neurons malfunction, however, the detection of leptin and the subsequent reduction in intake could be defective.

Another mechanism possibly contributing to the observed obesity phenotype involves adipogenesis. $B b s 1-4, B b s 6-8, B b s 9$, and $B b s 11$ are all expressed during mouse adipogenesis (76), suggesting they may play a role in the generation of fat tissue. Other ciliary proteins have also been implicated in this process, including retinitis-pigmentosa GTPase regulator interacting protein 1-like (RPGRIP1L), which localizes to the basal body (77) and whose expression is decreased in the adipose tissue of mutants for the adjacent FTO gene (78) and the Alström syndrome gene ALMS1, for which obese knockout mice have been generated $(79,80)$. The ALMS1 protein, which localizes to the basal body $(81,82)$, is expressed in the early phases of adipogenesis and may be involved in the conversion of preadipocytes to adipocytes (83). It remains to be determined whether hypothalamic dysfunction alone is sufficient to induce obesity in BBS and other ciliopathies. The attenuated phenotype of the POMC:Cre Ift 88 and Kif $3 a$ mutants 
can be explained either by the fact that some leptinergic neurons might have escaped inactivation or that there is a systemic contribution. In addition, it will be important to measure energy expenditure in ciliary mouse mutants and human patients, since a contribution of defective sensing of energy expenditure cannot yet be excluded. Ultimately, systematic tissue-specific ablation and crossing of the mutant animals will be required to answer this question comprehensively.

Polydactyly. Polydactyly in BBS and other ciliopathies is intriguing because of the known mechanisms of digit formation and the involvement of SHH signaling. Digit formation starts in the zone of polarizing activity (ZPA), a structure in the posterior mesenchyme of the limb (or fin) bud that is common in vertebrates (S25). SHH is found in the vertebrate limb bud, including the zebrafish fin bud, and regulates the $\mathrm{ZPA}$ (S26, S27). The targets of SHH signaling are the glioma (GLI) transcription factors GLI1, GLI2, and GLI3 (84). When SHH binds to the Patched1 (PTCH1) receptor, Smoothened (SMO) is derepressed, blocking processing of GLI3 from its activator to repressor forms (S28). In the context of limb formation, $\mathrm{SHH}$ regulates digit number and identity (S29), and either ectopic $\mathrm{SHH}$ expression or loss of GLI3 causes polydactyly (S30-S32).

Several lines of evidence implicate the cilium in SHH signaling and digit formation, not least of which is the localization of both SMO and PTCH1 to the cilium, where PTCH1 inhibits SMO by preventing its accumulation at the cilium $(68,69)$. SHH binding of PTCH1 causes it to be mislocalized from the cilium so that SMO can be activated there (69) (Figure 2). IFT protein function is required for SHH signaling. Mouse mutants for $1 f t 172$ and Ift88 exhibit phenotypes consistent with defects in SHH signaling, including loss of ventral neural cell populations and preaxial polydactyly (85). The defect appears to be downstream of PTCH1 and SMO, possibly at the level of GLI processing. A later study confirmed that the defect lies in the proteolytic processing of GLI3 to its repressor form, which requires IFT172 (86). Another study of Ift 88 mutants revealed that GLI2 and GLI3 - in addition to a negative regulator of $\mathrm{SHH}$, Sufu - also localize to the cilium in the developing limb bud and require IFT88 to do so (87).

Additional findings support the role of IFT in Shh signaling: IFT proteins regulate both activator and repressor Gli expression (88), and suppression of retrograde IFT results in mislocalization of SMO from the cilium and disruption of Gli3 processing (89). It also appears that the interaction of SMO and the IFT protein kinesin family member $3 A$ (KIF3A) is regulated by $\beta$-arrestin (90). Loss of KIF3A in cartilage results in skeletogenic defects (91), consistent with the role of IFT in regulating limb formation, possibly through $\mathrm{SHH}$. Taken together, these findings provide strong evidence that IFT may regulate Shh signaling in limb bud cells and that defects in this regulation results in aberrant formation of digits.

BBS proteins have been linked to Shh regulation of limb development as well. Expression of $b b s 7$ is enriched in the developing zebrafish fin bud (32). Furthermore, exogenous misexpression of either $b b s 1$ or $b b s 7$ results in increased Shh expression in the anterior ZPA and skeletal pattern changes in the pectoral fin consistent with a link between BBS proteins and Shh-directed limb development (92). It is notable, however, that the polydactyly associated with BBS is almost always postaxial. While other disorders associated with postaxial polydactyly, notably Pallister-Hall syndrome (S33), arise from defects in Shh signaling, the possibility exists that at least one other signaling pathway is involved, the Wnt pathway. This is because mice lacking Dkk1, an extracellular protein that binds the LRP5/6 receptor to antagonize canonical Wnt signaling, also exhibit postaxial polydactyly, as do hypomorphic Dkk1 mutants $(93,94)$. Loss of BBS protein function results in defects in suppression of noncanonical Wnt signaling, with a slight increase in targets of canonical Wnt signaling (66). Thus, the suppression of BBS proteins may have the net result of producing defects similar to those seen in mice with upregulation of canonical Wnt signaling.

Renal dysfunction. The low incidence of renal function abnormalities in BBS patients and mouse models, relative to other features of the disorder, has made this phenotype one of the less well-investigated aspects of disease. However, other ciliopathies exhibit a range of renal phenotypes (Figure 3), which, in addition to reports of kidney defects in ciliary mouse mutants, provides some insight into the mechanisms underlying the renal phenotype. Notably, the hypomorphic Ift88 mouse mutant orpk provided a model for PKD as a result of defective cilia assembly (95). Other ciliary proteins have been associated with kidney disease as well (reviewed in ref. S34).

Several lines of evidence support a role for BBS proteins, including the direct interaction of BBS1, $-2,-4$, and -7 with proteins present in the kidney (96). Bbs4-null mice display shorter renal tubule cilia initially, and these become longer over time, indicating a defect of either cilia assembly (97) or maintenance/regulation of ciliary length. Compelling evidence implicating BBS proteins in kidney phenotypes was exhibited with the formation of kidney cysts in bbs zebrafish morphants (98). Interestingly, the cyst phenotype could be rescued by culturing embryos in a solution containing the mTOR signaling inhibitor rapamycin (98). These findings provide support for a possible role for pathways upstream of mTOR in the kidney phenotype, most notably the noncanonical Wnt planar cell polarity (PCP) pathway.

BBS proteins, as well as other ciliary proteins, have been implicated in PCP signaling. Suppression of BBS protein function in mice or zebrafish, for example, produces defects reminiscent of the classical phenotypes resulting from mutations in PCP genes (including Vangl2), such as neural tube defects, open eyelids, perturbation of cochlear stereociliary bundles, and disruption of convergent extension movements (99). Furthermore, BBS genes interact with core PCP mutants, underscoring their role, and the role of cilia, as key regulators of the pathway. Further investigation revealed the interaction of BBS proteins with noncanonical Wnt ligands (Wnt5 and Wnt11), which, when perturbed, produces convergent extension defects and stabilization of cytoplasmic levels of $\beta$-catenin as a result of defective proteasome function (66). This defect may be linked to IFT function, as it is phenocopied by suppression of kif3a (66), which suppresses canonical Wnt signaling by blocking the casein kinase-mediated phosphorylation of the scaffold protein dishevelled (67). Interestingly, disruption of PCP produces defects in ciliogenesis as well. Perturbation of the PCP effectors inturned and fuzzy results in defects in ciliogenesis and convergent extension as a result of the interaction with dishevelled at the basal body (100). These findings are consistent with a role of cilia in regulation of PCP signaling and vice versa (Figure 2 ).

This is particularly relevant to the renal disease phenotype because the PCP pathway regulates cell polarity and orientation during the development of the nephron; cell mitotic spindles must be oriented properly during proliferation and tubule extension (101). Cilia have been implicated in this process, not least because loss of cilia in kidney, as induced by Kif3a knockout specific to renal tubular epithelial cells via Ksp:Cre, demonstrated that loss of PCP- 

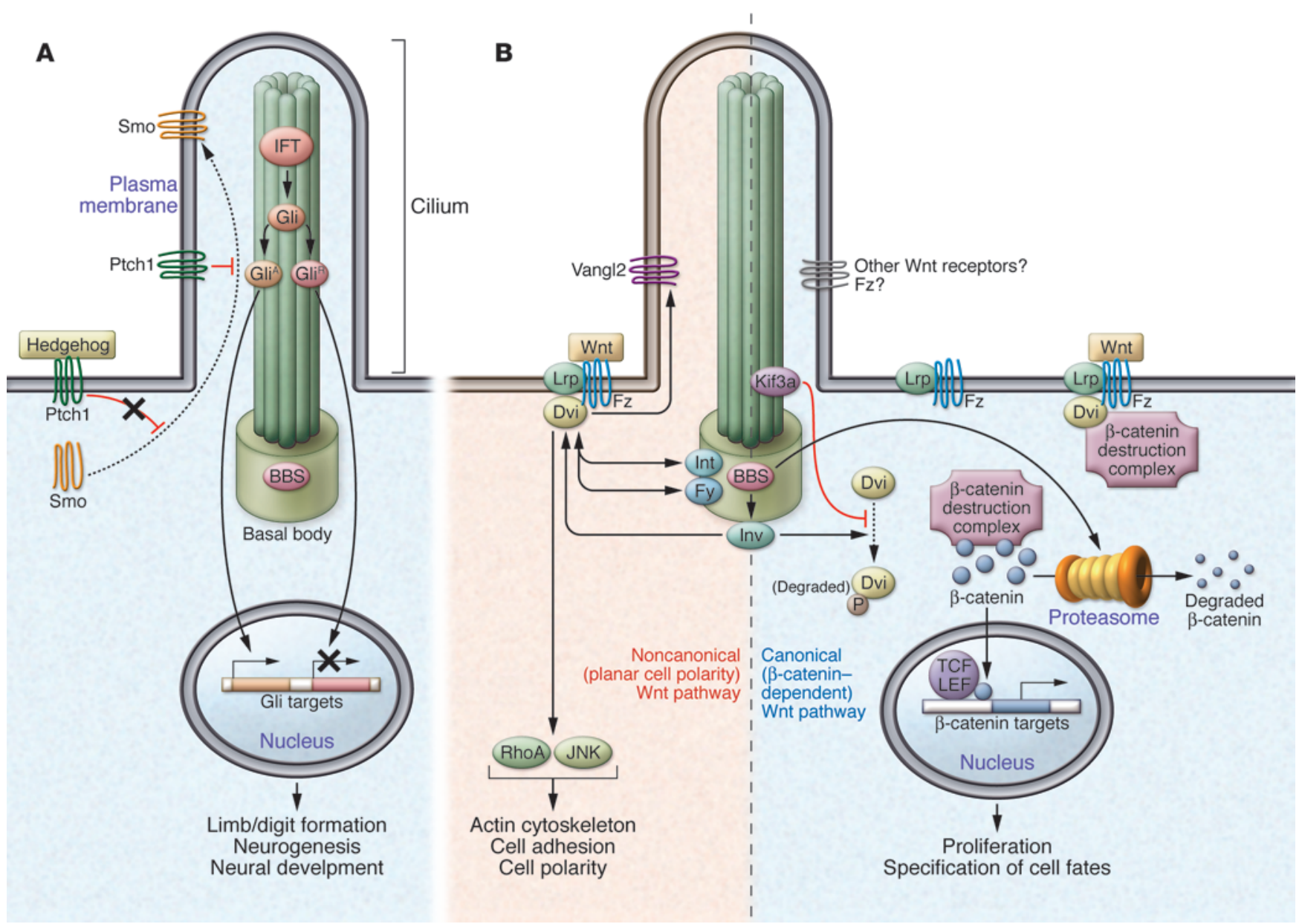

\section{Figure 2}

Signaling pathways in the cilium. (A) Hedgehog signaling is regulated by the Ptch1 and Smo receptors. Binding of the hedgehog ligand to Ptch1 alleviates its inhibition of transport of Smo into the cilium, where it may regulate IFT involvement in Gli processing to either its activator form (Gli ${ }^{A}$ ) or repressor form $(\mathrm{Gli})$. Downstream targets of Gli-directed transcription include limb/digit formation, neurogenesis, and neural development. (B) Canonical and noncanonical Wnt signaling components are associated with the cilium and basal body. Binding of a canonical Wnt ligand to Frizzled (Fz) and LPL-related protein (Lrp) receptors causes Dishevelled-mediated (Dvl-mediated) recruitment of the $\beta$-catenin destruction complex to the plasma membrane, preventing $\beta$-catenin degradation. $\beta$-Catenin can then regulate T cell factor-and lymphoid-enhanced binding factor-mediated (TCF- and LEF-mediated) transcription of targets in the nucleus and subsequent processes such as cell proliferation and specification of cell fates. In the absence of ligand, Dvl and the $\beta$-catenin destruction complex exist freely in the cytoplasm and can target $\beta$-catenin for proteasome-mediated degradation. BBS proteins regulate proteasome function in addition to the transport of Inversin (Inv) from the basal body into the cytoplasm, where it can reduce cytoplasmic levels of Dvl via phosphorylation, a process that can also be regulated by Kif3a. Binding of a noncanonical Wnt ligand also recruits Dvl to the Fz receptor, a process regulated by Inv. This initiates activation of downstream targets, including the PCP effectors RhoA and JNK, and downstream processes that affect the actin cytoskeleton, cell adhesion, and cell polarity. Regulation of PCP signaling also occurs at the level of Dvl and its interactions with Vangl2 in the cilium and inturned (Int) and fuzzy (Fy) at the basal body.

dependent mitotic spindle orientation produces cysts as a result of proliferation defects (102). Similarly, ablation of Ift20 in collecting duct cells produces cystic kidneys as a result of failure of proliferating cells to properly orient their mitotic spindles along the tubule (103). Another model of PCP defects, the Fat4-null mouse, also forms cystic kidneys as a result of aberrant mitotic spindle orientation (104). These findings are consistent with the finding that loss of $P k h d 1$, a basal body protein associated with autosomal recessive PKD (ARPKD) (105), disrupts PCP signaling (101).

In addition to the association of PCP defects with renal pathology, $\beta$-catenin-dependent canonical Wnt signals are widely believed to be important for kidney development. Wnt9b and Wnt4 have been implicated in the specification of epithelial cells to a renal fate $(106,107)$, and treatment of isolated rat kidney mesenchyme with the GSK3- $\beta$ inactivators lithium or 6-bromoindirubin-3'oxime induces nephron differentiation (108), indicating that excessive $\beta$-catenin promotes the process. This has also been demonstrated in vivo in mice, where conditional loss of $\beta$-catenin in kidney targeted to ureteric cells results in defects in branching morphogenesis and renal dysplasia (109).

There is evidence that regulation of the canonical Wnt pathway is linked to the cilium, especially with respect to kidney phenotypes. For example, cells of the developing mouse kidney lacking cilia as a result of conditional Ift20 knockout, which leads to cystic kidneys, 

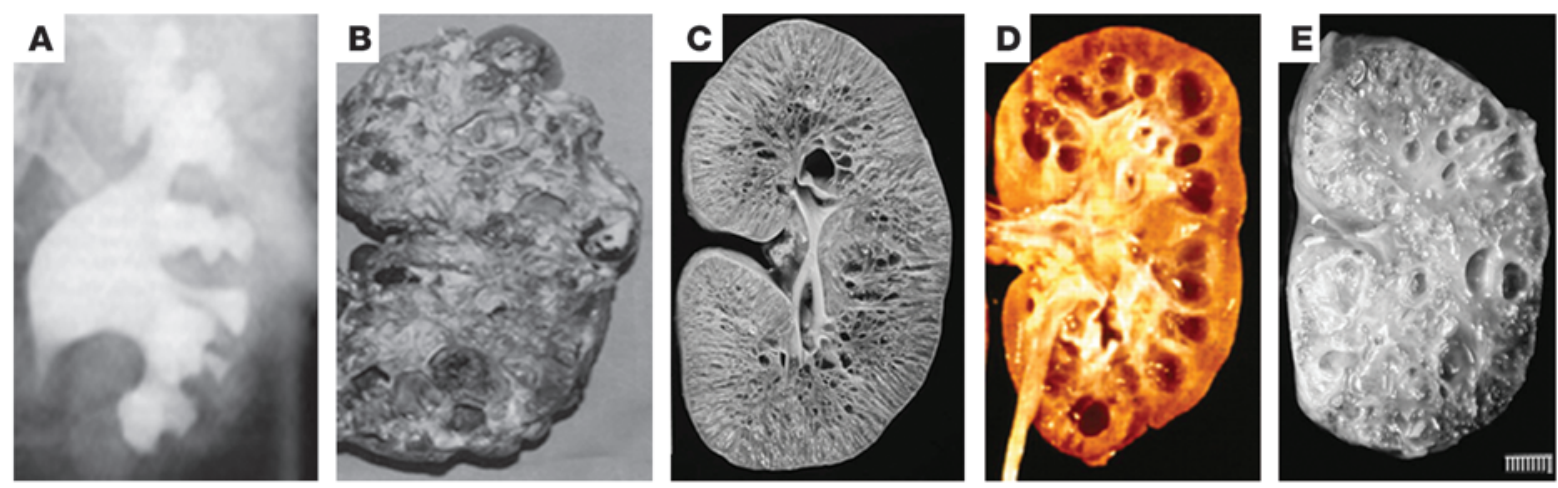

Figure 3

Renal phenotypes of ciliopathies. (A) Intravenous pyelogram of BBS kidney showing pyelocalyceal dilatation, calyceal clubbing, and pericalyceal cysts. (B) ADPKD kidneys typically have numerous cysts of variable sizes, with even distribution throughout the renal cortex and medulla. (C) ARPKD kidneys retain a reniform configuration with radiating, fusiform nonobstructive dilatations of the collecting ducts extending from the medulla to the cortex. (D) In NPH - as in JBTS, Senior-Loken Syndrome, and Jeune syndrome - the kidney exhibits cysts arising from the corticomedullary junction. (E) MKS kidneys manifest cystic dysplastic changes. Images reproduced with permission from Radiology (125) (A), Journal of the American Society of Nephrology (113) (D), and Advances in Anatomic Pathology (126) (B, C, and E). Scale bar: $1 \mathrm{~cm}$.

exhibit increased intracellular canonical Wnt signaling (103). Consistent with this, in the zebrafish, loss of seahorse (human homolog: LRCC6) results in cystic kidneys as a result of its interaction with the ciliary gene inversin and regulation of dishevelled (110). This is important in the context of Wnt signaling because inversin seems to function as a switch between canonical and noncanonical Wnt regulation in the kidney (111) (Figure 2). It is possible, then, that cilia regulate the dissemination of Wnt signaling in the cell, which directs downstream targets to drive cell fate specification and morphogenesis in the developing kidney.

The role of cilia in Wnt signaling suggests that defects in this pathway underlie the overlapping kidney phenotypes across the ciliopathies. However, there are distinct differences in both quality and severity of defects across ciliopathies (Figure 3). For example, reports of kidney phenotypes in BBS patients have included hyperplasia, dysplasia, and cystic kidneys (112). Other disorders, however, exhibit only hyperplasia and severely cystic kidneys (ARPKD) or progressive dysplasia with localized cysts (NPH) $(33,113)$. Furthermore, some ciliopathies, such as AS and NPH, manifest other phenotypes, such as fibrosis. The varying severity and presentation of additional phenotypes suggest that proteins underlying these disorders may either have additional cellular functions or participate in different components of the same pathways. Regarding the first possibility, one example lies in the fact that upregulation of growth factors, such as EGF and TGF, is thought to contribute to the hyperplasia and cysts associated with ARPKD (114); the involvement of TGF also suggests a link to the fibrosis phenotype (115). If either hypothesis proves to be true, this would implicate ciliary proteins in regulation of growth factor pathways at the cellular level, though a direct link has yet to be demonstrated. Finally, with respect to the differential severity of perturbation of intracellular pathways by perturbation of ciliary proteins, the link between BBS and NPH may shed light onto the varying severity produced by targeting a signaling pathway differentially. One protein underlying NPH is inversin (NPHP2), which targets dishevelled for degradation to prevent $\beta$-catenin-dependent proliferation (111). Mutations in inversin may partially affect its function; however, mutations in BBS disrupt retrograde IFT and, subsequently, may disrupt transport of inversin to the cytoplasm altogether, completely preventing its interaction with dishevelled (112). Thus, the specific step disrupted in a pathway could dictate the severity of the resulting cellular, and physiologic, defect.

Mental retardation. One of the least-understood ciliopathy phenotypes is mental retardation. Behavioral abnormalities have been reported in Bbs-knockout mice, but the underlying physical defect has not been explored in detail. Though the exact function of ciliary proteins in brain development is unclear, expression of at least one BBS gene, Bbs3/Arl6, which is involved in ciliary transport (25, $116)$, is seen in developing neural tissues (117). Furthermore, consistent with cerebral anomalies observed in BBS patients (118), the Bbs1-M390R knock-in mouse had several morphological defects in the brain, including ventriculomegaly of the lateral and third ventricles, a thin cerebral cortex, and reduced corpus striatum hippocampus (53). Additionally, cilia along the enlarged third ventricle, though intact, were elongated and swollen at the distal end, suggestive of IFT defects.

There is evidence linking cilia to two processes in neural development: neurogenesis and neuronal migration. Recent evidence has linked Shh signaling, regulated by the cilium, to neurogenesis and hippocampal development. Conditional ablation of Kif $3 a$ in hGFAP:Cre cells results in defects in postnatal neurogenesis in the dentate gyrus, and this process is apparently regulated by Smo at the cilium, as conditional hGFAP:Cre;Smo mutants show similar defects, and constitutively active Smo was not able to rescue the conditional Kif3a phenotype (119). Furthermore, loss of cilia due to specific ablation of the ciliary gene Stumpy in hippocampal astrocyte-like neural precursors results in gross morphological abnormalities as a result of defects in neuronal precursor proliferation and defective Shh signaling (120). The involvement of Shh signaling at the cilium in proliferation of developing neuronal populations provides strong evidence that defective cilia play an important role in properly populating the hippocampus.

In addition to the generation of neurons during development, the migration of those neurons to various regions is also affected in BBS and potentially other ciliary mutants. BBS modulation of Wnt signaling may play a role in regulating movement of neuronal precursors. Loss of the zebrafish PCP protein strabismus $(s t b m)$, in Trilobite mutants results in defects in cell polarity and result- 
ing cell movements, including migration of hindbrain neurons (121). Similar migration defects result in suppression of another PCP protein, Prickle1, which interacts with stbm (122). Similarly, BBS proteins interact with stbm in Trilobite embryos, suggesting that this interaction plays a role in BBS neuronal phenotypes (99). The involvement of BBS proteins and the cilium in regulation of PCP signaling suggests that cilia may play a role in cell movements during development and, specifically, the migration of neurons during brain development.

\section{Concluding remarks}

Since the cloning of the first gene for BBS in $2000(123,124)$, the field has witnessed significant progress in elucidating both the genetic basis of the disorder and its underlying molecular defects. Moreover, the association of BBS with ciliary dysfunction has contributed to the expansion of our appreciation of the complex signaling mechanisms these structures partake in, while at the same time facilitating the integration of clinically discrete clinical entities into a common continuum of causality.

These findings have potentially important ramifications for the management and treatment of the disorder. A clinical diagnostic test covering all 14 known BBS genes is likely to have sensitivity of $50 \%-75 \%$ (depending on patient ancestry). However, the predictive power of the genotype will remain limited until the genetic basis of the phenotypic variability is understood. At present, prognoses of endophenotypes are based on epidemiology; ascertainment of mutational information to predict the expected rate of retinal degeneration, or which patients might require renal transplantation, for example, is not yet feasible. Even though genotype-phenotype correlations under a recessive model have failed in BBS, we predict that a model of total mutational load of the system (namely ciliary signaling) holds promise, not only for BBS but for other ciliopathies as well.

Regarding treatment, our present understanding of the molecular basis of ciliopathies suggests that discrete pathways might exert a major effect in discrete organ systems. For example, $\mathrm{SHH}$ signaling defects may underlie polydactyly and cognitive impairment, whereas aberrant Wnt signaling ( $\beta$-catenin dependent or independent) is a likely major driver of some of the renal manifes- tations. Thus, the possibility of a generalized therapeutic regime seems unrealistic, with the possible exception of gene replacement therapy (since the defect is likely caused by loss of function). From a pharmacological perspective, one would predict that an organspecific approach might prove useful. Indeed, the rapamycin treatment of the zebrafish cystic BBS model (98) suggests that mTOR targeting might be of therapeutic benefit. However, it is unclear whether, and probably unlikely that, this approach will be useful in postmitotic tissues, such as the brain and the retina.

Importantly, the phenotypic, genetic, and biochemical overlap between BBS and other ciliopathies suggests that any therapeutic approaches have the potential to benefit a broad spectrum of clinical phenotypes and an increased number of patients; although each ciliopathy is individually rare, collectively they represent a clinical continuum with a frequency exceeding 1:1,000 live births. Thus, therapeutic intervention in any given ciliary disease is likely to have broad application, potentially overcoming some of the socioeconomic hurdles associated with treatment of rare genetic disorders.

\section{Acknowledgments}

We apologize to those colleagues whose work we have been unable to cite due to a restriction on the number of citations. We thank Jose Badano and Erica Davis for their critical review of the manuscript and all the members of the Katsanis laboratory for helpful discussions. We also thank Meral Gunay-Aygun for compiling the images in Figure 3. This work was supported by grants from the National Institute of Child Health and Development (R01HD04260), the National Institute of Diabetes, Digestive, and Kidney Disorders (R01DK072301 and R01DK075972), and the Macular Vision Research Foundation (to N. Katsanis); and by a Visual Neuroscience Training Program fellowship (to N.A. Zaghloul).

Address correspondence to: Nicholas Katsanis, Johns Hopkins University School of Medicine, Suite 439, Broadway Research Building, 733 North Broadway, Baltimore, Maryland 21205, USA. Phone: (410) 502-6660; Fax: (410) 502-0697; E-mail: katsanis@jhmi.edu.
1. Klein, D., and Ammann, F. 1969. The syndrome of Laurence-Moon-Bardet-Biedl and allied diseases in Switzerland. Clinical, genetic and epidemiological studies. J. Neurol. Sci. 9:479-513.

2. Beales, P.L., Warner, A.M., Hitman, G.A., Thakker, R., and Flinter, F.A. 1997. Bardet-Biedl syndrome: a molecular and phenotypic study of 18 families. J. Med. Genet. 34:92-98.

3. Farag, T.I., and Teebi, A.S. 1988. Bardet-Biedl and Laurence-Moon syndromes in a mixed Arab population. Clin. Genet. 33:78-82

4. Farag, T.I., and Teebi, A.S. 1989. High incidence of Bardet Biedl syndrome among the Bedouin. Clin. Genet. 36:463-464.

5. Laurence, J., and Moon, R.C. 1866. Four cases of retinitis pigmentosa occurring in the same family accompanied by general imperfection of development. Ophthalmic Rev. 2:32-41.

6. Bardet, G. 1920. Sur un syndrome d'obesite congenitale avec polydactylie et retinite pigmentaire (contribution a l'etude des formes cliniques de l'obesite bypophysaire). Univ. de Paris. Thesis no. 470. Amedee LeGrand. Paris, France.

7. Biedl, A. 1922. Ein Geschwisterpaar mit adiposo-genitaler Dystrophie. Dtsch. Med. Wochenschr. 48:1630.

8. Green, J.S., et al. 1989. The cardinal manifesta- tions of Bardet-Biedl syndrome, a form of Laurence-Moon-Biedl syndrome. N. Engl. J. Med. 321:1002-1009.

9. Beales, P.L., Elcioglu, N., Woolf, A.S., Parker, D., and Flinter, F.A. 1999. New criteria for improved diagnosis of Bardet-Biedl syndrome: results of a population survey. J. Med. Genet. 36:437-446.

10. Kulaga, H.M., et al. 2004. Loss of BBS proteins causes anosmia in humans and defects in olfactory cilia structure and function in the mouse. Nat. Genet. 36:994-998.

11. Tan, P.L., et al. 2007. Loss of Bardet Biedl syndrome proteins causes defects in peripheral sensory innervation and function. Proc. Natl. Acad. Sci. U. S. A. 104:17524-17529.

12. Leitch, C.C., et al. 2008. Hypomorphic mutations in syndromic encephalocele genes are associated with Bardet-Biedl syndrome. Nat. Genet. 40:443-448.

13. Mykytyn, K., et al. 2003. Evaluation of complex inheritance involving the most common BardetBiedl syndrome locus (BBS1). Am. J. Hum. Genet. 72:429-437.

14. Katsanis, N. 2004. The oligogenic properties of Bardet-Biedl syndrome. Hum. Mol. Genet. 13:R65-R71.

15. Stoetzel, C., et al. 2006. BBS10 encodes a vertebratespecific chaperonin-like protein and is a major BBS locus. Nat. Genet. 38:521-524.
16. Chiang, A.P., et al. 2006. Homozygosity mapping with SNP arrays identifies TRIM32, an E3 ubiquitin ligase, as a Bardet-Biedl syndrome gene (BBS11). Proc. Natl. Acad. Sci. U. S. A. 103:6287-6292.

17. Katsanis, N., et al. 2001. Triallelic inheritance in Bardet-Biedl syndrome, a Mendelian recessive disorder. Science. 293:2256-2259.

18. Badano, J.L., et al. 2006. Dissection of epistasis in oligogenic Bardet-Biedl syndrome. Nature. 439:326-330.

19. Katsanis, N., et al. 2002. BBS4 is a minor contributor to Bardet-Biedl syndrome and may also participate in triallelic inheritance. Am. J. Hum. Genet. 71:22-29.

20. Badano, J.L., et al. 2003. Heterozygous mutations in BBS1, BBS2 and BBS6 have a potential epistatic effect on Bardet-Biedl patients with two mutations at a second BBS locus. Hum. Mol. Genet. 12:1651-1659.

21. Beales, P.L., et al. 2003. Genetic interaction of BBS1 mutations with alleles at other BBS loci can result in non-Mendelian Bardet-Biedl syndrome. Am. J. Hum. Genet. 72:1187-1199.

22. Badano, J.L., and Katsanis, N. 2002. Beyond Mendel: an evolving view of human genetic disease transmission. Nat. Rev. Genet. 3:779-789.

23. Ansley, S.J., et al. 2003. Basal body dysfunction is a 
likely cause of pleiotropic Bardet-Biedl syndrome. Nature. 425:628-633.

24. Li, J.B., et al. 2004. Comparative genomics identifies a flagellar and basal body proteome that includes the BBS5 human disease gene. Cell. 117:541-552.

25. Fan, Y., et al. 2004. Mutations in a member of the Ras superfamily of small GTP-binding proteins causes Bardet-Biedl syndrome. Nat. Genet. 36:989-993.

26. Kim, J.C., et al. 2004. The Bardet-Biedl protein BBS4 targets cargo to the pericentriolar region and is required for microtubule anchoring and cell cycle progression. Nat. Genet. 36:462-470.

27. Kim, J.C., et al. 2005. MKKS/BBS6, a divergent chaperonin-like protein linked to the obesity disorder Bardet-Biedl syndrome, is a novel centrosomal component required for cytokinesis. J. Cell Sci. 118:1007-1020

28. Nachury, M.V., et al. 2007. A core complex of BBS proteins cooperates with the GTPase Rab8 to promote ciliary membrane biogenesis. Cell. 129:1201-1213.

29. Nonaka, S., et al. 1998. Randomization of leftright asymmetry due to loss of nodal cilia generating leftward flow of extraembryonic fluid in mice lacking KIF3B motor protein. Cell. 95:829-837.

30. Blacque, O.E., et al. 2004. Loss of C. elegans BBS-7 and BBS- 8 protein function results in cilia defects and compromised intraflagellar transport. Genes Dev. 18:1630-1642.

31. Ou, G., Blacque, O.E., Snow, J.J., Leroux, M.R., and Scholey, J.M. 2005. Functional coordination of intraflagellar transport motors. Nature. 436:583-587.

32. Yen, H.J., et al. 2006. Bardet-Biedl syndrome genes are important in retrograde intracellular trafficking and Kupffer's vesicle cilia function. Hum. Mol. Genet. 15:667-677.

33. Badano, J.L., Mitsuma, N., Beales, P.L., and Katsanis, N. 2006. The ciliopathies: an emerging class of human genetic disorders. Annu. Rev. Genomics Hum. Genet. 7:125-148.

34. Gherman, A., Davis, E.E., and Katsanis, N. 2006 The ciliary proteome database: an integrated community resource for the genetic and functional dissection of cilia. Nat. Genet. 38:961-962.

35. Kyttälä, M., et al. 2006. MKS1, encoding a component of the flagellar apparatus basal body proteome, is mutated in Meckel syndrome. Nat. Genet. 38: $155-157$.

36. Smith, U.M., et al. 2006. The transmembrane protein meckelin (MKS3) is mutated in MeckelGruber syndrome and the wpk rat. Nat. Genet. 38:191-196.

37. Baala, L., et al. 2007. Pleiotropic effects of CEP290 (NPHP6) mutations extend to Meckel syndrome. Am. J. Hum. Genet. 81:170-179.

38. Karmous-Benailly, H., et al. 2005. Antenatal presentation of Bardet-Biedl syndrome may mimic Meckel syndrome. Am. J. Hum. Genet. 76:493-504.

39. Bergmann, C., et al. 2008. Loss of nephrocystin-3 function can cause embryonic lethality, MeckelGruber-like syndrome, situs inversus, and renalhepatic-pancreatic dysplasia. Am. J. Hum. Genet. 82:959-970.

40. Hoefele, J., et al. 2007. Evidence of oligogenic inheritance in nephronophthisis. J. Am. Soc. Nephrol. 18:2789-2795.

41. Tory, K., et al. 2007. High NPHP1 and NPHP6 mutation rate in patients with Joubert syndrome and nephronophthisis: potential epistatic effect of NPHP6 and AHI1 mutations in patients with NPHP1 mutations. J. Am. Soc. Nephrol. 18:1566-1575.

42. Kamiya, A., et al. 2008. Recruitment of PCM1 to the centrosome by the cooperative action of DISC1 and BBS4: a candidate for psychiatric illnesses. Arch. Gen. Psychiatry. 65:996-1006.
43. Ostrowski, L.E., et al. 2002. A proteomic analysis of human cilia: identification of novel components. Mol. Cell. Proteomics. 1:451-465.

44. Kim, J., Krishnaswami, S.R., and Gleeson, J.G. 2008. CEP290 interacts with the centriolar satellite component PCM-1 and is required for Rab8 localization to the primary cilium. Hum. Mol. Genet. 17:3796-3805

45. Calarco-Gillam, P.D., Siebert, M.C., Hubble, R., Mitchison, T., and Kirschner, M. 1983. Centrosome development in early mouse embryos as defined by an autoantibody against pericentriolar material. Cell. 35:621-629.

46. Dawe, H.R., et al. 2007. The Meckel-Gruber Syndrome proteins MKS1 and meckelin interact and are required for primary cilium formation. Hum. Mol. Genet. 16:173-186.

47. Williams, C.L., Winkelbauer, M.E., Schafer, J.C., Michaud, E.J., and Yoder, B.K. 2008. Functional redundancy of the $\mathrm{B} 9$ proteins and nephrocystins in Caenorhabditis elegans ciliogenesis. Mol. Biol. Cell. 19:2154-2168.

48. Ammann, F. 1970. Investigations clinique et génétique sur le syndrome de Bardet-Biedl en Suisse. J. Genet. Hum. 18(Suppl.):1-310.

49. Nishimura, D.Y., et al. 2004. Bbs2-null mice have neurosensory deficits, a defect in social dominance, and retinopathy associated with mislocalization of rhodopsin. Proc. Natl. Acad. Sci. U. S. A. 101:16588-16593.

50. Fath, M.A., et al. 2005. Mkks-null mice have a phenotype resembling Bardet-Biedl syndrome. Hum. Mol. Genet. 14:1109-1118.

51. Eichers, E.R., et al. 2006. Phenotypic characterization of Bbs 4 null mice reveals age-dependent penetrance and variable expressivity. Hum. Genet. 120:211-226

52. Mykytyn, K., et al. 2004. Bardet-Biedl syndrome type 4 (BBS4)-null mice implicate Bbs4 in flagella formation but not global cilia assembly. Proc. Natl. Acad. Sci. U. S. A. 101:8664-8669.

53. Davis, R.E., et al. 2007. A knockin mouse model of the Bardet-Biedl syndrome 1 M390R mutation has cilia defects, ventriculomegaly, retinopathy, and obesity. Proc. Natl. Acad. Sci. U. S. A. 104:19422-19427.

54. Gerth, C., Zawadzki, R.J., Werner, J.S., and Heon, E. 2008. Retinal morphology in patients with BBS1 and BBS10 related Bardet-Biedl Syndrome evaluated by Fourier-domain optical coherence tomography. Vision Res. 48:392-399.

55. Malm, E., et al. 2008. Full-field electroretinography and marked variability in clinical phenotype of Alstrom syndrome. Arch. Ophthalmol. 126:51-57.

56. Hanein, S., et al. 2004. Leber congenital amaurosis: comprehensive survey of the genetic heterogeneity, refinement of the clinical definition, and genotypephenotype correlations as a strategy for molecular diagnosis. Hum. Mutat. 23:306-317.

57. Abd-El-Barr, M.M., et al. 2007. Impaired photoreceptor protein transport and synaptic transmission in a mouse model of Bardet-Biedl syndrome. Vision Res 47:3394-3407.

58. Chang, B., et al. 2006. In-frame deletion in a novel centrosomal/ciliary protein CEP290/NPHP6 perturbs its interaction with RPGR and results in early-onset retinal degeneration in the $\mathrm{rd} 16$ mouse. Hum. Mol. Genet. 15:1847-1857.

59. Swiderski, R.E., et al. 2007. Gene expression analysis of photoreceptor cell loss in bbs4-knockout mice reveals an early stress gene response and photoreceptor cell damage. Invest. Ophthalmol. Vis. Sci. 48:3329-3340

60. Moritz, O.L., et al. 2001. Mutant rab8 Impairs docking and fusion of rhodopsin-bearing postGolgi membranes and causes cell death of transgenic Xenopus rods. Mol. Biol. Cell. 12:2341-2351.

61. Pazour, G.J., et al. 2000. Chlamydomonas IFT88 and its mouse homologue, polycystic kidney disease gene $\operatorname{tg} 737$, are required for assembly of cilia and flagella. J. Cell Biol. 151:709-718.

62. Pazour, G.J., et al. 2002. The intraflagellar transport protein, IFT88, is essential for vertebrate photoreceptor assembly and maintenance. J. Cell Biol. 157:103-113.

63. Tsujikawa, M., and Malicki, J. 2004. Intraflagellar transport genes are essential for differentiation and survival of vertebrate sensory neurons. Neuron. 42:703-716.

64. Krock, B.L., and Perkins, B.D. 2008. The intraflagellar transport protein IFT57 is required for cilia maintenance and regulates IFT-particle-kinesin-II dissociation in vertebrate photoreceptors. J. Cell. Sci. 121:1907-1915.

65. Luby-Phelps, K., Fogerty, J., Baker, S.A., Pazour, G.J., and Besharse, J.C. 2008. Spatial distribution of intraflagellar transport proteins in vertebrate photoreceptors. Vision Res. 48:413-423.

66. Gerdes, J.M., et al. 2007. Disruption of the basal body compromises proteasomal function and perturbs intracellular Wnt response. Nat. Genet. 39:1350-1360.

67. Corbit, K.C., et al. 2008. Kif3a constrains betacatenin-dependent Wnt signalling through dual ciliary and non-ciliary mechanisms. Nat. Cell Biol. 10:70-76.

68. Corbit, K.C., et al. 2005. Vertebrate Smoothened functions at the primary cilium. Nature. 437:1018-1021.

69. Rohatgi, R., Milenkovic, L., and Scott, M.P. 2007. Patched 1 regulates hedgehog signaling at the primary cilium. Science. 317:372-376.

70. Rahmouni, K., et al. 2008. Leptin resistance contributes to obesity and hypertension in mouse models of Bardet-Biedl syndrome. J. Clin. Invest. 118:1458-1467.

71. Berbari, N.F., Lewis, J.S., Bishop, G.A., Askwith, C.C., and Mykytyn, K. 2008. Bardet-Biedl syndrome proteins are required for the localization of $\mathrm{G}$ protein-coupled receptors to primary cilia. Proc. Natl. Acad. Sci. U. S. A. 105:4242-4246.

72. Mak, H.Y., Nelson, L.S., Basson, M., Johnson, C.D., and Ruvkun, G. 2006. Polygenic control of Caenorhabditis elegans fat storage. Nat. Genet. 38:363-368.

73. Davenport, J.R., et al. 2007. Disruption of intraflagellar transport in adult mice leads to obesity and slow-onset cystic kidney disease. Curr. Biol. 17:1586-1594.

74. Satir, P. 2007. Cilia biology: stop overeating now! Curr. Biol. 17:R963-R965.

75. Ma, X., Zubcevic, L., and Ashcroft, F.M. 2008. Glucose regulates the effects of leptin on hypothalamic POMC neurons. Proc. Natl. Acad. Sci. U. S. A. 105:9811-9816.

76. Forti, E., Aksanov, O., and Birk, R.Z. 2007. Temporal expression pattern of Bardet-Biedl syndrome genes in adipogenesis. Int. J. Biochem. Cell Biol. 39:1055-1062.

77. Vierkotten, J., Dildrop, R., Peters, T., Wang, B., and Ruther, U. 2007. Ftm is a novel basal body protein of cilia involved in Shh signalling. Development. 134:2569-2577.

78. Stratigopoulos, G., et al. 2008. Regulation of Fto/Ftm gene expression in mice and humans. Am. J. Physiol. Regul. Integr. Comp. Physiol. 294:R1185-R1196.

79. Arsov, T., et al. 2006. Fat aussie - a new Alstrom syndrome mouse showing a critical role for ALMS1 in obesity, diabetes, and spermatogenesis. Mol. Endocrinol. 20:1610-1622.

80. Collin, G.B., et al. 2005. Alms1-disrupted mice recapitulate human Alstrom syndrome. Hum. Mol. Genet. 14:2323-2333.

81. Li, G., et al. 2007. A role for Alstrom syndrome protein, alms1, in kidney ciliogenesis and cellular quiescence. PLoS Genet. 3:e8. 
82. Hearn, T., et al. 2005. Subcellular localization of ALMS1 supports involvement of centrosome and basal body dysfunction in the pathogenesis of obesity, insulin resistance, and type 2 diabetes. Diabetes. 54:1581-1587.

83. Romano, S., et al. 2008. Regulation of Alstrom syndrome gene expression during adipogenesis and its relationship with fat cell insulin sensitivity. Int. J. Mol. Med. 21:731-736

84. Ingham, P.W., and McMahon, A.P. 2001. Hedgehog signaling in animal development: paradigms and principles. Genes Dev. 15:3059-3087.

85. Huangfu, D., et al. 2003. Hedgehog signalling in the mouse requires intraflagellar transport proteins. Nature. 426:83-87.

86. Huangfu, D., and Anderson, K.V. 2005. Cilia and Hedgehog responsiveness in the mouse. Proc. Natl. Acad. Sci. U. S. A. 102:11325-11330.

87. Haycraft, C.J., et al. 2005. Gli2 and Gli3 localize to cilia and require the intraflagellar transport protein polaris for processing and function. PLoS Genet. 1:e53.

88. Liu, A., Wang, B., and Niswander, L.A. 2005. Mouse intraflagellar transport proteins regulate both the activator and repressor functions of Gli transcription factors. Development. 132:3103-3111.

89. May, S.R., et al. 2005. Loss of the retrograde motor for IFT disrupts localization of Smo to cilia and prevents the expression of both activator and repressor functions of Gli. Dev. Biol. 287:378-389.

90. Kovacs, J.J., et al. 2008. Beta-arrestin-mediated localization of smoothened to the primary cilium. Science. 320:1777-1781.

91. Koyama, E., et al. 2007. Conditional Kif3a ablation causes abnormal hedgehog signaling topography, growth plate dysfunction, and excessive bone and cartilage formation during mouse skeletogenesis. Development. 134:2159-2169.

92. Tayeh, M.K., et al. 2008. Genetic interaction between Bardet-Biedl syndrome genes and implications for limb patterning. Hum. Mol. Genet. 17:1956-1967.

93. Mukhopadhyay, M., et al. 2001. Dickkopf1 is required for embryonic head induction and limb morphogenesis in the mouse. Dev. Cell. 1:423-434.

94. MacDonald, B.T., Adamska, M., and Meisler, M.H. 2004. Hypomorphic expression of Dkk1 in the doubleridge mouse: dose dependence and compensatory interactions with Lrp6. Development 131:2543-2552.

95. Cano, D.A., Murcia, N.S., Pazour, G.J., and Hebrok, M. 2004. Orpk mouse model of polycystic kidney disease reveals essential role of primary cilia in pancreatic tissue organization. Development. 131:3457-3467.
96. Oeffner, F., et al. 2008. Novel interaction partners of Bardet-Biedl syndrome proteins. Cell. Motil. Cytoskeleton. 65:143-155.

97. Mokrzan, E.M., Lewis, J.S., and Mykytyn, K. 2007. Differences in renal tubule primary cilia length in a mouse model of Bardet-Biedl syndrome. Nephron Exp. Nephrol. 106:e88-e96.

98. Tobin, J.L., and Beales, P.L. 2008. Restoration of renal function in zebrafish models of ciliopathies. Pediatr. Nephrol. 23:2095-2099.

99. Ross, A.J., et al. 2005. Disruption of Bardet-Biedl syndrome ciliary proteins perturbs planar cell polarity in vertebrates. Nat. Genet. 37:1135-1140.

100.Park, T.J., Haigo, S.L., and Wallingford, J.B. 2006 Ciliogenesis defects in embryos lacking inturned or fuzzy function are associated with failure of planar cell polarity and Hedgehog signaling. Nat. Genet. 38:303-311.

101.Fischer, E., and Pontoglio, M. 2006. Planar cell polarity and polycystic kidney disease [In French]. Med. Sci. (Paris). 22:576-578.

102. Patel, V., et al. 2008. Acute kidney injury and aberrant planar cell polarity induce cyst formation in mice lacking renal cilia. Hum. Mol. Genet. 17:1578-1590.

103.Jonassen, J.A., San Agustin, J., Follit, J.A., and Pazour, G.J. 2008. Deletion of IFT20 in the mouse kidney causes misorientation of the mitotic spindle and cystic kidney disease. J. Cell Biol. 183:377-384.

104.Saburi, S., et al. 2008. Loss of Fat4 disrupts PCP signaling and oriented cell division and leads to cystic kidney disease. Nat. Genet. 40:1010-1015.

105. Ward, C.J., et al. 2002. The gene mutated in autosomal recessive polycystic kidney disease encodes a large, receptor-like protein. Nat. Genet. 30:259-269.

106. Lyons, J.P., et al. 2004. Wnt-4 activates the canonical beta-catenin-mediated Wnt pathway and binds Frizzled-6 CRD: functional implications of Wnt/ beta-catenin activity in kidney epithelial cells. Exp. Cell Res. 298:369-387.

107. Carroll, T.J., Park, J.S., Hayashi, S., Majumdar, A., and McMahon, A.P. 2005. Wnt9b plays a central role in the regulation of mesenchymal to epithelial transitions underlying organogenesis of the mammalian urogenital system. Dev. Cell. 9:283-292.

108.Kuure, S., Popsueva, A., Jakobson, M., Sainio, K., and Sariola, H. 2007. Glycogen synthase kinase-3 inactivation and stabilization of beta-catenin induce nephron differentiation in isolated mouse and rat kidney mesenchymes. J. Am. Soc. Nephrol. 18:1130-1139.

109. Bridgewater, D., et al. 2008. Canonical WNT/betacatenin signaling is required for ureteric branching. Dev. Biol. 317:83-94.

110.Kishimoto, N., Cao, Y., Park, A., and Sun, Z. 2008. Cystic kidney gene seahorse regulates cilia- mediated processes and Wnt pathways. Dev. Cell. 14:954-961.

111.Simons, M., et al. 2005. Inversin, the gene product mutated in nephronophthisis type II, functions as a molecular switch between Wnt signaling pathways. Nat. Genet. 37:537-543.

112.Tobin, J.L., and Beales, P.L. 2007. Bardet-Biedl syndrome: beyond the cilium. Pediatr. Nephrol. 22:926-936.

113.Hildebrandt, F., and Zhou, W. 2007. Nephronophthisis-associated ciliopathies. J. Am. Soc. Nephrol. 18:1855-1871.

114.Sweeney, W.E., Jr., and Avner, E.D. 2006. Molecular and cellular pathophysiology of autosomal recessive polycystic kidney disease (ARPKD). Cell Tissue Res. 326:671-685.

115.Torres, V.E., et al. 2003. EGF receptor tyrosine kinase inhibition attenuates the development of PKD in Han:SPRD rats. Kidney Int. 64:1573-1579.

116.Chiang, A.P., et al. 2004. Comparative genomic analysis identifies an ADP-ribosylation factor-like gene as the cause of Bardet-Biedl syndrome (BBS3). Am. J. Hum. Genet. 75:475-484.

117. Takada, T., Iida, K., Sasaki, H., Taira, M., and Kimura, H. 2005. Expression of ADP-ribosylation factor (ARF)-like protein 6 during mouse embryonic development. Int. J. Dev. Biol. 49:891-894.

118. Rooryck, C., et al. 2007. Bardet-Biedl syndrome and brain abnormalities. Neuropediatrics. 38:5-9.

119. Han, Y.G., et al. 2008. Hedgehog signaling and primary cilia are required for the formation of adult neural stem cells. Nat. Neurosci. 11:277-284.

120.Breunig, J.J., et al. 2008. Primary cilia regulate hippocampal neurogenesis by mediating sonic hedgehog signaling. Proc. Natl. Acad. Sci. U. S. A. 105:13127-13132.

121.Jessen, J.R., et al. 2002. Zebrafish trilobite identifies new roles for Strabismus in gastrulation and neuronal movements. Nat. Cell Biol. 4:610-615.

122.Carreira-Barbosa, F., et al. 2003. Prickle 1 regulates cell movements during gastrulation and neuronal migration in zebrafish. Development. 130:4037-4046.

123. Slavotinek, A.M., et al. 2000. Mutations in MKKS cause Bardet-Biedl syndrome. Nat. Genet. 26:15-16.

124. Katsanis, N., et al. 2000. Mutations in MKKS cause obesity, retinal dystrophy and renal malformations associated with Bardet-Biedl syndrome. Nat. Genet. 26:67-70.

125.Alton, D.J., and McDonald, P. 1973. Urographic findings in the Bardet-Biedl syndrome, formerly the Laurence-Moon-Biedl syndrome. Radiology. 109:659-663.

126.Bisceglia, M., et al. 2006. Renal cystic diseases: a review. Adv. Anat. Pathol. 13:26-56. 